\title{
An unexpected complication in a hemodialysis patient: midodrine related $\mathrm{A}-\mathrm{V}$ fistula dysfunction
}

\author{
(D) Mehmet Emin Demir ${ }^{1}$, ĐÇağlar Alp ${ }^{2}$ \\ ${ }^{1}$ İstanbul Yeni Yüzyıl University, Gaziosmanpaşa Hospital, Department of Nephrology, İstanbul, Turkey \\ ${ }^{2}$ Kırıkkale University, Faculty of Medicine, Department of Cardiology, Kırıkkale, Turkey
}

Cite this article as: Demir ME, Alp Ç. An unexpected complication in a hemodialysis patient: midodrine related A-V fistula dysfunction. J Health Sci Med 2021; 4(3): 397-399.

\begin{abstract}
Arteriovenous fistula (AVF) dysfunction is one of the common obstacles in performing hemodialysis treatment. Acute and chronic thrombosis of the fistula, stenosis, and hematomas are the major causes of the AVF dysfunction. Here, we present an unexpected complication of midodrine use which caused in AVF dysfunction in a female patient who was suffering from hemodialysis-related hypotension.
\end{abstract}

Keywords: AV fistula, hemodialysis, midodrine

\section{INTRODUCTION}

Hypotension is a serious problem in hemodialysis patients and causes discomfort during hemodialysis (HD) sessions. It also causes HD inadequacy and may cause life-threatening complications. Dialysisassociated hypotension is usually associated with removing high volume in a relatively short time during an HD session. HD patients with diabetes mellitus, amyloidosis, and uremic neuropathy could have an autonomic dysfunction-caused hypotensive period which is not uncommon, in the course of the disease. Midodrine, an alpha-adrenergic agonist drug that increases arterial and venous system resistance, has off-label use in HD patients, in order to prevent orthostatic hypotension (1). It is an alternative drug preferred in the treatment of hemodialysis-related hypotension by most centers (2). Here, we present a 62-year-old female HD patient who developed an acute arteriovenous fistula (AVF) dysfunction after a recent commenced midodrine therapy for hemodialysisrelated hypotension.

\section{CASE}

A 62-year-old female HD patient was referred to our center on a suspicion of having venous stenosis in the AVF arm. Her past medical records revealed low KTV, high serum phosphorus and potassium levels, and loss of well-being which all were indicating an
HD inadequacy. Her pre-dialysis and intradialytic blood pressure levels were low and frequent medical interventions were complicating the $\mathrm{HD}$ sessions. In a physical examination, a well thrilling AVF with relatively not prominent venous dilatation was noted. The physician had advised her to receive $20 \mathrm{mg}$ of midodrine on hemodialysis days and $10 \mathrm{mg}$ on dialysisfree days within the last two weeks. She had also used midodrine in the past, however with lower doses, for the treatment of intradialytic hypotension. We tried to perform an HD session in our HD center, however, low blood flow and frequent venous line warnings due to high venous line pressure made it difficult to complete the HD session. Doppler ultrasound measured $615 \mathrm{ml} /$ min blood flow of the AVF. Fistulogram (venography) studies revealed diffuse vasospasm in the cephalic vein (Figure 1). We did not determine obvious stenosis throughout the cephalic vein. We administered a nitrate infusion protocol through the cephalic vein insertion point and diffuse vasospasm on the cephalic vein resolved (Figure 2 and Figure 3). The patient was advised to stop taking midodrine, henceforth. In follow-ups; HD sessions are being maintaned without any previously described symptoms and findings. 




Figure 1. Diffuse vasospasm in the cephalic vein



Figure 2. After $100 \mathrm{mcg}$ nitrate infusion



Figure 3. After $200 \mathrm{mc}$ nitrate infusion

\section{DISCUSSION}

Hemodialysis-related hypotension is a prevalent complication in the HD population. Modifying HD prescription, cessation of antihypertensive drugs (at least on HD days), not allowing eating during HD sessions may prevent the development of hypotension, however, this approach fails or is inadequate mostly. While some patients well tolerate dialysis-related hypotension many patients suffer from hypotension and are not able to complete an optimal HD session as desired. Alphaagonists constrict both arteries and veins; however, the vasoconstrictor effect is more pronounced in the arterial resistance vessels. Constriction of the resistance vessels (small arteries and arterioles) increases systemic vascular resistance, whereas constriction of the venous capacitance vessels increases venous pressure (1).

Alpha-1 and alpha-2 adrenoceptor agonists cause venoconstriction in vivo, but alpha 2-receptor-mediated constriction is intrinsically weaker. Midodrine is an alpha-1 adrenergic agonist and considered in the treatment of orthostatic hypotension. The drug has a usage field in hemodialysis-related hypotension, as a off-label drug. Observational, randomized controlled, and crossover studies have shown the benefits of the drug on systolic and diastolic blood pressure, and also improvement of symptoms of intradialytic hypotension. Additionally, midodrine use has not been associated with late fistula failure in HD patients, by today (3).

Midodrine related adverse reactions;

- Cardiovascular: Supine hypertension (7-13\%)

- Centraal nervous system: Paresthesia (18\%)

- Dermatologic: Piloerection (13\%), pruritus (12\%)

- Genitourinary: Dysuria $(<13 \%)$, urinary retention, and urgency

- Polyuria, chills, skin rash, abdominal pain, leg cramps...

also has been reported.

Midodrine-induced adverse reactions are generally mild and transient, responding to a decrease in midodrine dosage. It is believed that using midodrine is safe and effective in hemodialysis patients who suffer from hypotension. But long-term effects are not clear, particularly on the cardiovascular system. Arteriovenous fistula dysfunction is a challenging obstacle in hemodialysis practice in the term of vascular access. Venous thrombosis, stenosis, and strictures are the most common causes of the dysfunction. The dysfunction results in hemodialysis inadequacy and should be corrected as soon as possible (2). 
In our case, despite lack of severe arterial blood pressure increase (mean pressures 135/80 $\mathrm{mmHg}$, however higher levels than before midodrine treatment), venous spasm confirmed on the arterio-venous fistula arm by using a venogram. Glyceryl trinitrate (Perlinganite) administration resolved venoconstriction, and cessation of midodrine prevented relapse of high venous line pressure during hemodialysis. Thus, recirculation was removed and KTV improved.

We aimed to report this extraordinary complication which we linked to midodrine use, and make clinicians keep in mind to reassess all medications when encountering a dialysis-related complication.

\section{ETHICAL DECLARATIONS}

Informed Consent: Written informed consent was obtained from all participants who participated in this study.

Referee Evaluation Process: Externally peer-reviewed.

Conflict of Interest Statement: The authors have no conflicts of interest to declare.

Financial Disclosure: The authors declared that this study has received no financial support.

Author Contributions: All of the authors declare that they have all participated in the design, execution, and analysis of the paper, and that they have approved the final version.

\section{REFERENCES}

1. Böchl-Daum B, Korn A, Wolzt M, Schmidt E, Eichler HG. In vivo studies on alpha-adrenergic receptor subtypes in human veins. Naunyn Schmiedebergs Arch Pharmacol 1991; 344: 302-7.

2. Prakash S, Garg AX, Heidenheim P, House AA. Midodrine appearrs to be safe and effective for dialysis-induced hypotension a systematic review. Nephrol Dial Transplant 2003; 19: 2553-8.

3. Yen CC, Tsai CF, Luo YY, Yang HY, Liu MY, Hung PH, Hsu YH. Factors affecting fistula failure in patients on chronic hemodialysis: a population-based case-control study. BMC Nephrol. 2018; 19: 213. 